\title{
The investigation of the coefficients of materials of hard hulls of autonomous deep-underwater vehicles
}

\author{
Aleksandr Karpachev* \\ Chair of the Mathematics Department, Pacific Higher Naval College, Vladivostok, Russia
}

\begin{abstract}
The conceptional projecting of new type of marine technique presented by autonomous underwater vehicles based on new type of materials for hard hulls. As is known, the quality of the materials of hard hulls depends on the Poisson coefficient and the modulus of elasticity (Young), which play an important role in calculating the critical pressure for stability. The problem of finding the optimum values of these coefficients (the maximum of the critical pressure) is of great interest for the creation of ideal materials for the hard hulls of underwater vehicles. Such a problem must be solved in conjunction with the task of optimizing another coefficient - the material's fluidity module. In the Investigations it is very important to accurately calculate the critical pressure for the stability of the hard hulls of underwater vehicles. This method of exact calculation has been created and tested in practice for known materials: aluminum alloy, steel, titanium and fiberglass. The method was also used to solve the problem of creating a new type of material for hard hulls with optimal values of the yield coefficients, Jung and Poisson.
\end{abstract}

\section{Introduction}

As is known, for the design of autonomous underwater vehicles (AUV), a technology is used, in which one large hard hull (or container) is used, forming the middle, cylindrical part of the apparatus and ensuring its buoyancy. Lightweight extremities filled with water are attached to the container, where part of the payload of the AUV, made in the submersible version, is located.

The task of designing a hard hull of optimum design, i.e., having sufficient strength at minimum weight, for underwater vehicles depends on the quality of the materials of manufacture. In turn, the quality of the materials of the hard hulls depends on the Poisson's ratio $(E)$ and the modulus of elasticity $(m u)$, which play an important role in calculating the critical pressure for stability.

\section{The finding the optimal cylindrical hull}

\footnotetext{
*Corresponding author: alaf1978@yandex.ru
} 
The problem of finding the optimum values of these coefficients (the maximum of the critical pressure) is of great interest for the creation of ideal materials for hard hulls of underwater vehicles. In most practical cases, the calculations of a hard hull are based on methods for calculating the strength and stability of cylindrical shells of rotation.

The thickness $d$ of the cylindrical part of the hull, determined from the strength formula, does not yet guarantee its destruction. The hydrostatic pressure $Q$, being external to the hull, causes its obshchatie. In this case, significant compressive stresses appear in the hull. Numerous experiments have shown that at voltages that occur at the calculated depth $H$, a loss of stability of the cylindrical body can occur, which will lead to its destruction.

Under these conditions, the parameters of the cylindrical hull must be determined in such a way that the magnitude of the critical pressure $P$ is not less than the value of the design pressure $Q$.

The problem of determining the stability of cylindrical hulls was solved in work [1]. In this case, smooth cylindrical hulls and hulls were considered separately, supported by transverse circular frames (external and internal). In each individual case, the corresponding formulas were obtained to determine the magnitude of the critical pressure.

To check the cylindrical hull for stability, it is necessary to calculate the critical pressure $P$, at which the hull does not change its shape, i.e. will not undergo any deformation. Comparison of the results of theoretical calculations of the masses of cylindrical hulls with the actual masses of the hard hulls of existing underwater vehicles shows that the error of the calculation formulas is up to $15 \%$ for the depth range from 300 to $1000 \mathrm{~m}$ and below.

For a AUV, this error is significant, so the task of developing a more accurate formula for calculating pressure on stability is important. As is known, to solve specific problems of calculating the strength and stability of hulls, the theory of calculations of axisymmetric bodies of revolution of arbitrary shape is used, based on assumptions about the homogeneity and isotropy of the materials of manufacture. Two ways of solving this problem are fundamentally possible: either in displacements, or in efforts and moments. In the first way, a system of three differential equations is used, expressed through forces and moments, which, in turn, are determined through the relative deformations of the middle surface.

After the deformation components are replaced by displacements of the middle surface of the hull, they arrive at a system of three differential equations with three unknown displacements $u, v, w$, whose integration under the given boundary conditions reduces the solution of the problem posed.

The second solution path involves the addition of three equations of continuity of the middle surface to the system of equations of equilibrium in forces and moments. Integration of the resulting system of equations with six unknown forces and moments for given boundary conditions reduces the solution of the problem of hull theory in forces and moments. As in the first and in the second cases, the system of equations has the eighth order, that is, it contains the partial differential equations of the eighth order. In view of the assumptions made, the system of equilibrium equations in displacements is transformed into a linear differential system. However, in a simplified linear formulation the problem remains very complicated.

\section{The exact solution for stability pressure}

In work [1], a differential system of equations was reduced to an algebraic system of equations containing the required critical pressure as an unknown quantity. This system has a solution, if its determinant is zero. Expanding this determinant and equating it to zero, a rather complicated third-order equation is obtained with respect to the parameter $P$. To reduce the order of this equation, quantities containing the quantities $P, E$ and $m u$ are 
usually neglected, accepting new agreements and simplifications. As a result, we obtain a relatively simple first-order equation, from which we find the practical formula for calculating the critical pressure $P$.

However, we can, without neglecting these quantities, using the properties of the determinant of the sum of two matrices, find the exact value of the pressure $P$ from the condition that the determinant of the system is zero. This exact solution with loss of stability of the hull depends on $m$ - the number of half-waves of the sinusoid along the length of the hull and $n$ - the number of half-waves along its perimeter. It is necessary to find a minimum of $P(m, n)$, varying the values of $m$ and $n$. Numerous computational experiments have shown that the pressure $P(m, n)$ for any values of $n$ and at practical values of the parameters $r$ and $l$ of the hull reaches a minimum at $m=1$, i.e. the hull loses stability with the formation of one half-wave along its length. In this case, the value of $n$ indicates the number of half-waves formed around the perimeter of the hull.

The solution of the problem of minimizing $P(1, n)$ is carried out by a simple search for $n=1,2,3, \ldots, N$, to the first value of $N$, for which

$$
P(1, N)<P(1, N+1)
$$

Busting does not take much time. The value of $N$, as a rule, does not exceed 20. It should be noted that in order to compensate for the effect on the stability of non-linear factors (the initial loss of the hull, material inhomogeneity, load pulsation, etc.), the value of the critical pressure can be corrected by some coefficient. The above method was implemented in the form of an algorithmic function

$$
P=E C P(l, r, d, E, m u)
$$

\section{The applications of the algorithmic function}

The algorithmic value of the critical pressure is used to test the case of thickness $d$ for stability. The pressure $Q$ can be represented as a function $Q=Q(d, H$, St), where $S t$ is the yield stress of the material of the hull. In the case that $P>Q$, the rugged case will be stable.

If $P<Q$, the hard hull will be unstable and need to be supported by transverse circular frames. To determine the required frame parameters, you can also use the function

$$
E C P(l, r, d, E, m u)
$$

It is important to note that for the fabrication of frames it is possible to use another type of material, i.e. with other values of the coefficients $E, m u$, St. To determine the number of frames, it is necessary to reduce the value of the length of the hard hull $l$ until the condition.

$$
\operatorname{ECP}(l ', r, d, E, m u)>Q
$$

where $l^{\prime}$ is the length of the hard hull between the frames. Then the number of necessary frames can be considered as a ratio $l / l^{\prime}$. Of course, this value must be rounded to the nearest integer and 1 more is added.

Note that this method additionally solves the problem of ensuring the strength and stability of the frame itself. The function $\operatorname{ECP}(l, r, d, E, m u)$ satisfies the universality condition, that is, it allows calculating both non-reinforced and supported strong cases.

The stability of strong rotating casings of arbitrary shape of constant thickness has been investigated by a number of authors [2-3]. The most exact solution of the problem of calculating the shells of revolution for an arbitrary load was obtained in complex form. It turns out that in this case the resulting differential equations admit a transformation, as a result of which the calculation of such a hard hull leads to the solution of a fourth-order 
differential system containing only two equations. From this system, the result can be obtained by integrating one equation of the second order [4,5].

This result can approximately be extended to rigid bodies of revolution of arbitrary shape. You can use the function $E C P(l, r, d, E, m u)$ with an average value of the radius $r$ along the entire length of this type of hull.

We construct a function

$$
F(l, r, d, E, m u, H, S t)=E C P(l, r, d, E, m u)-Q(d, H, S t)
$$

The problem of finding the optimum values of the coefficients of the material $E, m u, S t$, which provide the maximum of this function, is of great interest. Such values can be considered optimal for the creation of ideal materials for hard hulls of underwater vehicles.

It is important to note that additional conditions can be used to solve the task of optimizing a function, then it is necessary to compose the Lagrange function with additional conditions in mind. For example, you can require that condition

$$
F(l, r, d, E, m u, H, S t)<C O N S T
$$

For fixed values of the depth $H$, parameters $r$ and $l$ of a durable housing, it is also possible to determine the optimum values of the coefficients $E, m u$, St at the investigated ranges of their values.

\section{The investigation of the coefficients of materials}

The calculations showed that a change in the Poisson's ratio by 0.1 corresponds to a change in the critical pressure to $0.2 \mathrm{MPa}$ for a depth of $1000 \mathrm{~m}$ in some strong cases. This method of exact calculation has been created and tested in practice for known materials: aluminum alloy, steel, titanium and fiberglass.

So, for example, for a cylindrical hull made of fiberglass, with the parameters: $l=0,5 \mathrm{~m}$. and $H=3000 \mathrm{~m}$. The shell thickness $d=0.024 \mathrm{~m}$. is calculated, which provides a pressure strength $Q=45.1 \mathrm{MPa}$. However, when checking the stability of this hull, the formula for calculating the critical pressure determines a value that is greater than the indicated $P=56.0 M P a$. hard hull is stable.

As is known, increasing the competitiveness of promising marine and underwater means requires the use of fundamentally new structural materials with significantly improved performance characteristics. The world experience in the use of underwater vehicles proves that without them it is impossible to imagine the implementation of rescue and search operations, hydrographic and biological research, exploration of oil and gas fields, laying of main pipelines and installation of underwater oil storages, maintenance and repair of various underwater structures.

With the help of uninhabited underwater robotized vehicles, searches and inspections of sunken objects and artificial structures, exploration and mapping of the bottom, work in the construction and operation of hydroconstructions, submarine cables, pipelines and other facilities are carried out. The main and important problem of offshore development is the delivery of equipment for studying the seabed to great depths where it is impossible to use conventional diving equipment.

Typical trends for modern underwater vehicles are an increase in the depth of immersion and displacement. In connection with the growing demand for underwater work, new improved devices with an increased depth of immersion up to 6,000-7,000 $\mathrm{m}$ are being developed, new requirements are being introduced to the performance of the vehicles in terms of increasing their autonomy, speed and useful payload. With the expansion of the program of research and work in the World Ocean in various types of depressions and faults, which have not been investigated at the moment, the problem arises of creating 
apparatus for working at depths of more than 7,000 m, which requires further enhancement of the strength of hull materials.

For the manufacture of hard hulls of deep-water vehicles, materials with high specific strength, corrosion resistance and manufacturability are needed. To the greatest extent these requirements are met by titanium alloys. High-strength aluminum alloys also have high specific strength, but in comparison with titanium alloys have lower corrosion and technological properties.

The creation and use of structural composite materials is the dominant concept of the development of modern materials science. Experimental studies in this area show that fiberglass in terms of specific compressive strengths far exceeds such structural materials as steel, aluminum and titanium alloys. Silicate glasses achieve strength of about $10 \mathrm{GPa}$, much exceeding the strength of titanium alloys.

The high strength of fiberglass at a relatively low weight makes it possible to create unique structures of deepwater and rocket and space technology. Deep water vehicles will acquire the ability to work at the deepest depths of the World Ocean without using additional volumes of buoyancy. Fiberglass is a promising material for rugged hulls of underwater vehicles from the point of view of ensuring their strength, stability and production cost in comparison with the used in the present time for working at great depths of titanium alloy bodies [6].

Conclusion

The developed method of exact calculation of the critical pressure for the stability of cylindrical hulls, realized by the algorithmic function $E C P(l, r, d, E, m u)$ is adequate to existing practical formulas, and at the same time provides increased accuracy of calculations from 5 to $15 \%$ for various calculated depths. Accuracy increases with an increase in the calculated depth.

The algorithmic function $E C P(l, r, d, E, m u)$ does not require constraints on the ratios $l / r$, or any other constraints and assumptions, and is universal, i.e. it is applicable to the calculation of the critical pressure for the stability of hard hulls of containers and other underwater vehicles.

The algorithmic function $F(l, r, d, E, m u, H, S t)$ can also be used to solve the problem of creating a new type of material for strong casing with optimal values of the yield coefficients, Young and Poisson.

\section{References}

1. G. Illarionov, Problems of strength of ship structures under complex loading Vladivostok: , Far Eastern Federal University. (2016)

2. V. Pikul, Method for manufacturing a cylindrical shell of a durable underwater vehicle body made of fiberglass. - Vladivostok / IPMT Far Eastern Branch of the Academy of Sciences of the Russia, (2013)

3. V. Pikul, Method for manufacturing sheet three-layer fiberglass -Vladivostok/ IPMT Far Eastern Branch of the Academy of Sciences of the Russia, (2015)

4. A. Laptev, Additional requirements for long-range autonomous underwater vehiclesVladivostok/ IPMT Far Eastern Branch of the Academy of Sciences of the Russia, (2017)

5. G. Illarionov, Small-sized multifunctional autonomous unmanned underwater vehicle Vladivostok / IPMT Far Eastern Branch of the Academy of Sciences of the Russia, (2017)

6. Gonchruk V.K. Transport properties of solid solutions PbF2-SnF2-SbF3 prepared by solid state techniques // Solid State Ionics, (2017), Vol. 302 